\title{
Genomic analyses of microdissected Hodgkin and Reed-Sternberg cells: mutations in epigenetic regulators and p53 are frequent in refractory classic Hodgkin lymphoma
}

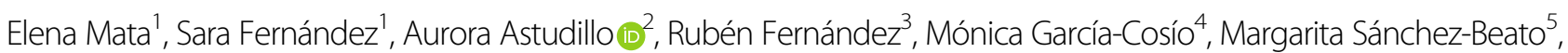 \\ Mariano Provencio ${ }^{5}$, Mónica Estévez ${ }^{6}$, Carlos Montalbán ${ }^{6}$, Miguel A. Piris ${ }^{7}$ and Juan F. García ${ }^{1}$
}

Classical studies have consistently identified some specific gene mutations in classic Hodgkin lymphoma (cHL), mainly affecting members of the NF-kappaB and JAK/ STAT pathways ${ }^{1-4}$. However, this knowledge is yet to be exploited in the clinical milieu. Molecular analyses of cHL have been limited because of the paucity of Hodgkin and Reed-Sternberg (HRS) cells, which usually account for $<5 \%$ of cells as identified by standard CD30 immunostaining. Various enrichment strategies, such as laser capture microdissection (LCM), could facilitate the identification of genetic alterations ${ }^{5}$. Also, in recent years, the sensitivity and specificity of next-generation sequencing (NGS) techniques have been greatly improved by simultaneously testing selected genes, arranged in comprehensive gene panels. This is even possible with formalin-fixed, paraffin-embedded (FFPE) tissue samples.

However, very few NGS studies have described the genomic landscape of the disease. The first whole-exome sequencing analyses of primary Hodgkin and ReedSternberg (HRS) cells found beta-2-microglobulin (B2M) to be the most commonly altered gene ${ }^{6}$. Recently, our group have identified concurrent genetic lesions in relevant signaling pathways, such as those of JAK-STAT, NFkappaB, and BCR, as well as in epigenetic regulators ${ }^{7}$. These findings are largely concordant with other NGS

\footnotetext{
Correspondence: Juan F. García (jfgarcia@mdanderson.es)

'Departments of Pathology, MD Anderson Cancer Center Madrid, Madrid,

Spain

${ }^{2}$ Departments of Pathology, Hospital Universitario de Cabueñes, Gijón, Spain Full list of author information is available at the end of the article.
}

studies in cHL cell lines ${ }^{8}$. More recently, Tiacci et $\mathrm{al}^{9}$ have reported frequent mutations affecting genes of the JAKSTAT pathway, as well as mutations in GNA13, XPO1, and ITPKB. However, none of these works set out to identify variants associated with response to treatment.

The identification of specific genetic lesions and better biological characterization of the subgroup of patients with refractory disease remain major research goals. Here we analyze the genomic characteristics of $12 \mathrm{cHL}$ tumors, corresponding to selected patients with diseases that are primary refractory to conventional therapy, and compare the genetic variants identified in the HRS cells from primary tumors and relapsed tumors.

Initially, formalin-fixed paraffin-embedded (FFPE) tumor samples and clinical data from $20 \mathrm{cHL}$ patients were obtained from the records of the participating institutions. All patients were intentionally selected because of their refractoriness to conventional treatment: primary-progressive disease (absence of complete remission after treatment) or early relapse (less than 12 months after complete remission). All cases received ABVD therapy. We collected representative tumor blocks from both, the original pretreatment and the relapse biopsy. All the samples and data were collected through the MDACC Madrid Biobank, in accordance with the technical and ethical procedures of the Spanish National Biobank Network, including anonymization processes and obtaining written informed consent according to the Helsinki Declaration. Approval was obtained from the institutional review board (Clinical Research Ethical Committee, ref. 
354/12). Clinical characteristics of the patients are summarized in Table 1. After LCM, DNA extraction and library construction, 12 cases fulfilled the minimum quality criteria and the generated libraries were sequenced and their data analyzed.

We enriched the tumor cells by LCM using a Palm MicroBeam V4 microscope (Carl Zeiss Inc., Oberkochen, Germany) equipped with a catapult system for contamination-free sample isolation. In brief, $5-\mu \mathrm{m}$ adjacent sections from FFPE tissues were cut, deparaffinized and immunostained with anti-CD30 antibody (clone BerH2, Dako/Agilent, Madrid, Spain). Parallel sections were also stained with hematoxylin and eosin to assess morphology, content and distribution of HRS cells.

All experimental procedures were repeated in the original pretreatment biopsy and the relapse biopsy, comparing each duplicated sample and discarding nonconcordant variants. From each case, 15,000-25,000 individually picked HRS cells duplicated per case were isolated from FFPE tissue. We also isolated normal lymphocytes from the reactive background (morphologically normal lymphocytes, CD30-negative, duplicated per case). This procedure was repeated in the original pretreatment biopsy and the relapse biopsy. Thus, eight different experiments were analyzed by NGS for each patient (all the workflow is depicted in Supplementary Figure 1).

After cell enrichment, we performed targeted analysis of 35 genes involved in B cell-related pathways (Supplemental Table 1), previously reported as being the most frequently mutated in $\mathrm{CHL}^{6,7,9}$, using Ion Torrent PGM (Thermo Fisher Scientific, NY, USA) technology with a modified protocol from that previously published ${ }^{7}$. DNA was extracted from duplicates of isolated HRS cells and CD30-negative fractions using the Gentra Puregene Tissue Kit (Qiagen, Germantown, MD). Libraries were constructed starting with $10 \mathrm{ng}$ of genomic DNA and following the manufacturer's protocol. Sequencing (BAM) files have been deposited in the NCBI Sequence Repository (SRA: PRJNA506444). The data were analyzed with the Torrent Suite program. All variants were examined with Integrative Genomics Viewer (IGV) software $^{10}$, discarding non-concordant variants. Functional consequences of the SNVs were predicted using the publicly available PROVEAN (shift and polyphen-2) and CONDEL algorithms.

Using this protocol for targeted sequencing, and after filtering SNPs, non-concordant, and silent variants, 42 candidate somatic SNVs were identified in the CD30positive fraction and 15 in the CD30-negative fraction, from the 238 initial candidate variants (Fig. 1 and Supplementary Table 2 summarize the results after comparing each duplicated sample and discarding nonconcordant variants). These figures could be related to genetic instability ${ }^{11}$, extensive clonal diversity and

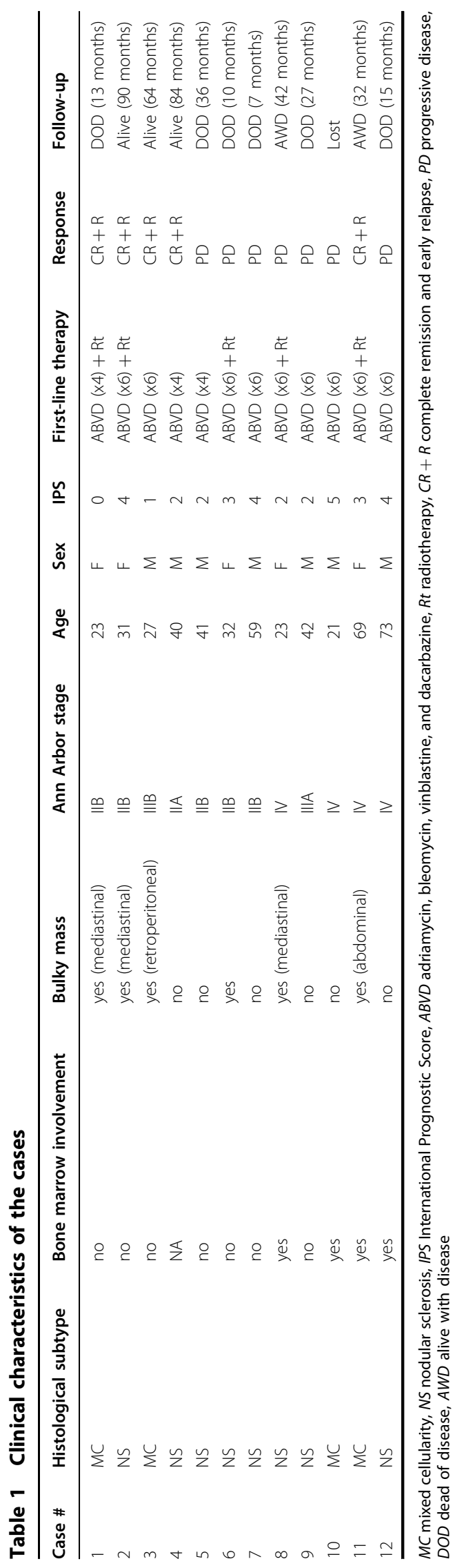




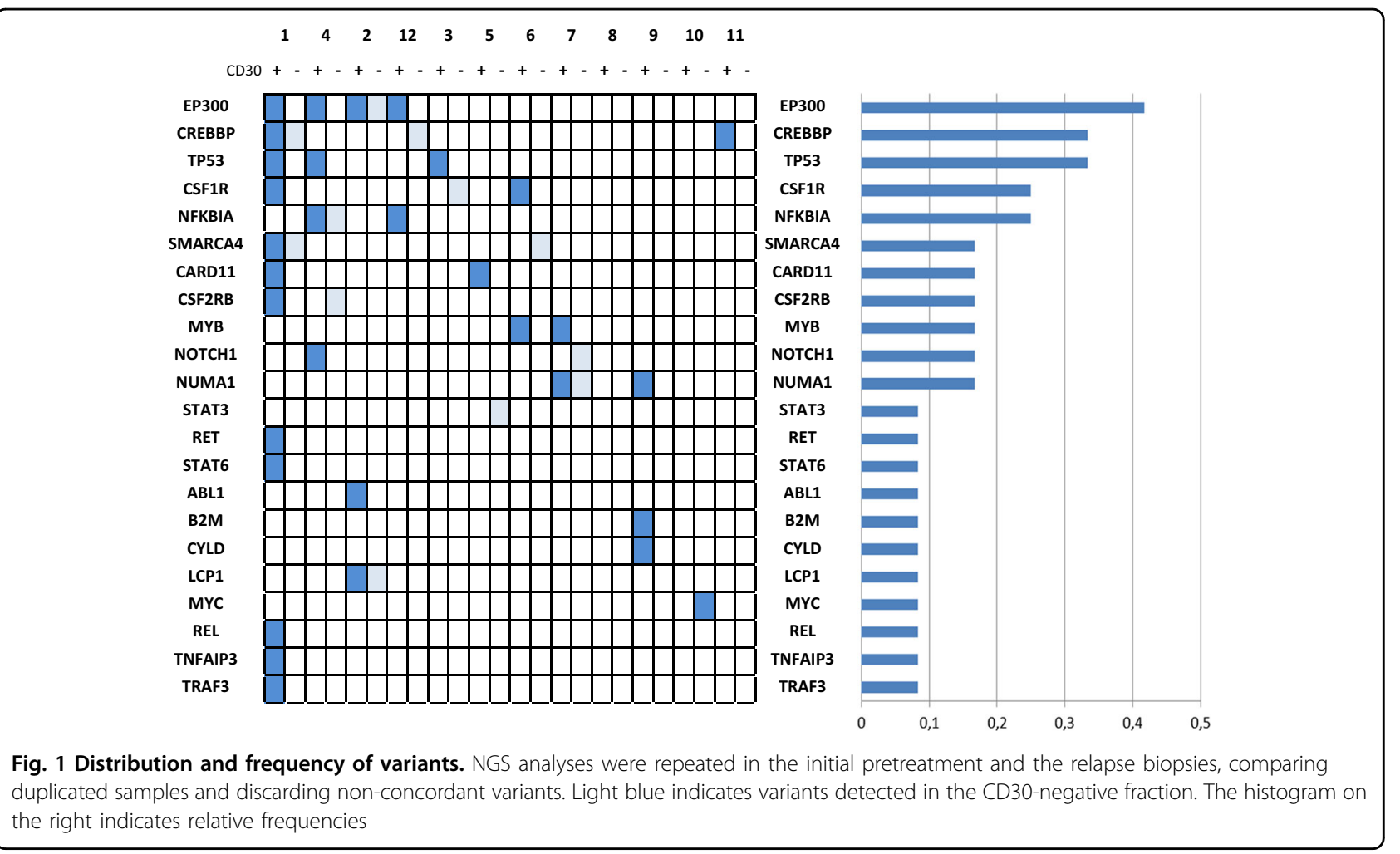

mutations with very low variant allele frequencies, as described in HRS cells ${ }^{7}$ and tumors ${ }^{9}$, or could arise because some of the individual variants are sequencing errors. An astringent filtering process, and the fact that, in all cases, the analysis was done in duplicate, guarantee that the risk of false-positive results is very low, even at the cost of losing valuable information about lowfrequency mutations and intratumor heterogeneity.

Most cases presented gene variants that had previously been described in $\mathrm{CHL}^{6,7,9}$. We confirmed the previously reported prevalent mutations affecting the NF-kappaB pathway, associated with JAK/STAT activation, $B 2 M$ mutations, and mutations affecting the BCR pathway.

Interestingly, mutations affecting the TP53 gene were overrepresented in this series of refractory cases ( 3 out of 12 cases, $25 \%$ ), with some variants that are redundantly detected in different cases, such as C238Y (cases 1 and 3) or Y234* (cases 3 and 4). These results contrast with classic studies that focused solely on p53 pathway mutations in $\mathrm{cHL}$, and previous NGS studies of unselected $\mathrm{cHL}$ only identified rare TP53 mutations (Supplementary Table 3). It is worth noting that TP53 mutations have been more frequently described recently in refractory $\mathrm{cHL}$ through the use of more sensitive NGS techniques ${ }^{12}$. This is entirely unsurprising since mutations of the p53 pathway are a classic prognostic factor associated with chemoresistance in most cancers.
In addition, the most frequently mutated genes, EP300 and $C R E B B P$, correspond to epigenetic regulators. Identical variants were detected in different cases and also in the CD30-positive and CD30-negative fractions, such as EP300 N1776H or CREBBP P1083L. EP300 encodes a transcriptional coactivator protein and functions as a histone acetyltransferase and regulates transcription via chromatin remodeling. Confirming previous results, mutations in the histone acetylation domains of EP300 and $C R E B B P$ are frequent in $\mathrm{cHL}$ (one or both alterations are present in 5 out of 12 cases, $41.6 \%$ ), similar to what is seen in DLBCL and follicular lymphoma. Furthermore, a recent report has presented evidence that histone acetyltransferase might act as a tumor suppressor that controls MHCII expression and promote tumor immune control and evasion ${ }^{13}$, and that drugs targeting histone acetyltransferase activities and chromatin remodeling, such as BET inhibitors, represent a novel therapeutic approach that have recently demonstrated efficacy in solid malignancies and lymphomas.

This study is obvious limited by the small number of cases, experimental complexity, and the impossibility of analyzing a parallel cohort of samples of "good responders" as control patients. Nevertheless, it seems clear that mutational frequencies are different from the distribution reported in previous unselected series (Supplementary Table 3). However, in a very recent study using a targeted 
NGS panel commercially available in archival tumor samples particularly enriched with relapsed patients, the most commonly mutated gene was also TP53, detected in 11 patients (22\%). Possible comparisons are limited by the obvious methodological differences between the various studies.

In our previous work ${ }^{7}$, in which $34 \%$ of the cases were primary refractory $\mathrm{cHL}, E P 300$ was also one of the most frequently mutated genes (occurring in $14 \%$ of cases). There were no statistically differences between mutational patterns of responders and non-responders in the original analyses, probably due to the small sample size. Nevertheless, further Kaplan-Meier analyses of failure-free survival (FFS), using the records from the 46 patients for whom we have complete clinical information and follow-up, revealed a significant association between the presence of CREBBP mutations and FFS (Supplemental Figure 2). Although we must recognize that a formal comparison between these different studies has obvious limitations.

An important observation in our study is the discovery of several mutations in the CD30-negative fraction in most of the experiments, using an LCM protocol with a very low probability of being subject to common contamination. We can speculate that these results might indicate a clonally related population, in a stem cell or less differentiated state, that is not morphologically or phenotypically distinguishable, as other authors have previously suggested ${ }^{14}$. In any case, additional experiments are necessary to corroborate this finding and fully understand its pathogenic significance.

In conclusion, it seems that several recurrent mutational events are present in primary refractory $\mathrm{cHL}$ that could be used as biomarkers and eventually exploited for therapy. TP53 mutations seem to represent a relevant predictive marker also in $\mathrm{cHL}$, and the highly prevalent mutations of epigenetic regulators EP300 and CREBBP suggest a rationale for alternative therapeutic strategies that need to be investigated further.

\section{Acknowledgements}

This work was supported by grants from the Plan Nacional de I + D + I cofinanced by the ISCIII-Subdirección General de Evaluación and the Fondo Europeo de Desarrollo Regional (FEDER), PI12/1832, the Spanish Association for Cancer Research (AECC), and Programas para Grupos de Investigación de la Comunidad Autónoma de Madrid (Biomedicina 2017). MSB currently holds a Miguel Servet II contract (CPII6/00024) supported by ISCIII-MINECO AESFEDER (Plan Estatal I + D + I 2013-2016) and the Fundación de Investigación Biomédica Puerta de Hierro.

\section{Author details}

'Departments of Pathology, MD Anderson Cancer Center Madrid, Madrid, Spain. ${ }^{2}$ Departments of Pathology, Hospital Universitario de Cabueñes, Gijón, Spain. ${ }^{3}$ Departments of Hematology, Hospital Universitario de Cabueñes, Gijón,
Spain. ${ }^{4}$ Department of Pathology, Hospital Universitario Ramón y Cajal, Madrid, Spain. ${ }^{5}$ Instituto Investigación Sanitaria Puerta de Hierro - Segovia de Arana (IDIPHISA), Centro de Investigación Biomédica en Red de Cáncer (CIBERONC) Instituto de Salud Carlos III, Madrid, Spain. ${ }^{6}$ Departments of Hematology, MD Anderson Cancer Center Madrid, Madrid, Spain. ${ }^{7}$ Department of Pathology, Fundación Jiménez Díaz, Madrid; Centro de Investigación Biomédica en Red de Cáncer (CIBERONC) Instituto de Salud Carlos III, Madrid, Spain

\section{Author contributions}

E.M. designed and performed the experiments and the targeted NGS panel. S. F. analyzed the sequencing data. A.A., R.F., M.G.-C., M.P., and M.E. provided clinical and diagnostic expertise and contributed to data interpretation. M.S.-B., C.M., M.A.P., and J.F.G. designed the experiments, directed the research, and wrote the manuscript.

\section{Conflict of interest}

The authors declare that they have no conflict of interest.

\section{Publisher's note}

Springer Nature remains neutral with regard to jurisdictional claims in published maps and institutional affiliations.

Supplementary Information accompanies this paper at (https://doi.org/ 10.1038/s41408-019-0195-7).

Received: 19 December 2018 Revised: 12 February 2019 Accepted: 25 February 2019

Published online: 11 March 2019

\section{References}

1. Emmerich, F. et al. Inactivating I kappa B epsilon mutations in Hodgkin/ReedSternberg cells. J. Pathol. 201, 413-420 (2003).

2. Schmitz, R. H. et al. TNFAIP3 (A20) is a tumor suppressor gene in Hodgkin lymphoma and primary mediastinal B cell lymphoma. J. Exp. Med. 206, 981-989 (2009).

3. Weniger, M. A. et al. Mutations of the tumor suppressor gene SOCS-1 in classical Hodgkin lymphoma are frequent and associated with nuclear phospho-STAT5 accumulation. Oncogene 25, 2679-2684 (2006).

4. Liu, X. et al. Mutations of NFKBIA in biopsy specimens from Hodgkin lymphoma. Cancer Genet. Cytogenet. 197, 152-157 (2010).

5. Tiacci, E. et al. Analyzing primary Hodgkin and Reed-Sternberg cells to capture the molecular and cellular pathogenesis of classical Hodgkin lymphoma. Blood 120, 4609-4620 (2012).

6. Reichel, J. et al. Flow sorting and exome sequencing reveal the oncogenome of primary Hodgkin and Reed-Sternberg cells. Blood 125, 1061-1072 (2015).

7. Mata, E. et al. Analysis of the mutational landscape of classic Hodgkin lymphoma identifies disease heterogeneity and potential therapeutic targets. Oncotarget 8, 111386-111395 (2017).

8. Liu, Y. et al. The mutational landscape of Hodgkin lymphoma cell lines determined by whole-exome sequencing. Leukemia 28, 2248-2251 (2014).

9. Tiacci, E. et al. Pervasive mutations of JAK-STAT pathway genes in classical Hodgkin lymphoma. Blood 131, 2454-2465 (2018).

10. Thorvaldsdóttir, H., Robinson, J. T. \& Mesirov, J. P. Integrative Genomic Viewer (IGV): high-performance genomics data visualization and exploration. Brief. Bioinform. 14, 178-192 (2012).

11. Re, D., Zander, T., Diehl, V. \& Wolf, J. Genetic instability in Hodgkin's lymphoma. Ann. Oncol. 13(Suppl 1), 19-22 (2002).

12. Liang, W. S. et al. Comprehensive genomic profiling of hodgkin lymphoma reveals recurrently mutated genes and increased mutation burden. Oncologist 23, 1-10 (2018).

13. Hashwah, $\mathrm{H}$. et al. Inactivation of CREBBP expands the germinal center $B$ cell compartment, down-regulates $\mathrm{MHCll}$ expression and promotes DLBCL growth. Proc. Natl Acad. Sci. USA 114, 9701-9706 (2017).

14. Jones, R. J. et al. Circulating clonotypic B cells in classic Hodgkin lymphoma. Blood 113, 5920-5926 (2009). 